\title{
La investigación en estilos de vida en psicología de consumidor ${ }^{1}$
}

\section{Research into lifestyles in consumer psychology}

DOI: http://dx.doi.org/10.17981/cultedusoc.8.1.2017.04

Diana Onofre-Chaves ${ }^{2}$

Fundación Universitaria Konrad Lorenz

\section{Resumen}

El propósito de este estudio fue realizar una revisión sistemática de los artículos científicos publicados entre 2010 y 2015 en torno a los estilos de vida en el área de consumidor para establecer el estado

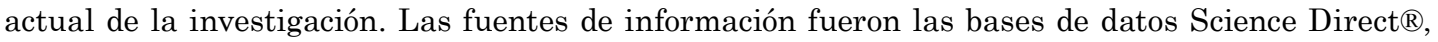
Springer Journal ${ }^{\circledR}$, Academic Search Complete ${ }^{\circledR}$ y Scopus ${ }^{\circledR}$. Un total de 94 artículos fueron depurados en una base de datos y se procedió a realizar análisis descriptivos. Se encontró una prevalencia de los estudios correlacionales y de tipo transversal. En la parte final del artículo se presentan los resultados de un análisis de convergencias que permite caracterizar los aspectos de interés crítico en el área. De acuerdo con el análisis de contenido realizado, se pueden definir los estilos de vida en el área de consumidor como una forma distintiva y consistente de vida, en la que están implicados procesos psicológicos, sociales y culturales. Estos procesos median en el proceso de compra, uso y descarte de bienes y servicios.

Palabras clave: Estilos de vida, revisión de literatura; psicología del consumidor; consumo conspicuo; consumo ideológico.

\footnotetext{
${ }^{1}$ Este proyecto fue financiado en la convocatoria 645 por el Departamento Administrativo de Ciencia y Tecnología e Innovación COLCIENCIAS

${ }^{2}$ Fundación Universitaria Konrad Lorenz. Correspondencia: dianac.onofrec@konradlorenz.edu.co
} 


\begin{abstract}
The purpose of this study was to carry out a systematic review of the scientific articles published between 2010 and 2015 around lifestyles in the consumer area to establish the current state of research. The sources of information were the Science Direct $\AA$, Springer Journal ${ }^{\circledR}$, Academic Search Complete ${ }^{\circledR}$ and Scopus ${ }^{\circledR}$ databases. A total of 94 articles were purified in a database and proceeded to perform descriptive analyzes. A prevalence of correlational and cross-sectional studies was found. In the final part of the article we present the results of a convergence analysis that allows us to characterize the aspects of critical interest in the area. According to the content analysis carried out, lifestyles can be defined in the consumer area as a distinctive and consistent way of life, in which psychological, social and cultural processes are involved. These processes mediate the process of purchase, use and disposal of goods and services.
\end{abstract}

Keywords: Lifestyles, literature review, consumer psychology, conspicuous consumption, ideological consumption.

\title{
La investigación en estilos de vida en psicología de consumidor
}

La literatura sobre el estilos de vida es extensa y aunque tiende a atribuirse al área del marketing, el término originalmente fue utilizado en ciencias sociales (Sarabia, Dolores De Juan y González, 2009). Las posturas iniciales concibieron los estilos de vida como las formas de ser, el estatus, la personalidad y el carácter del individuo (Daniela, Roxana y Elena, 2014; Lin, 2012). Posteriormente, el concepto se amplió en el sentido socio-cultural; el estilo de vida tiene un significado construido culturalmente y es un medio por el cual se establece la pertenencia a un grupo social particular y, en consecuencia, le permite a un individuo diferenciarse de los miembros de otros grupos (Sarabia, Dolores De Juan y González, 2009).

No hay una definición unívoca de los estilos de vida en la literatura científica (Schäfer, Jaeger-Erben, y Dos Santos, 2011). Sin embargo, existen múltiples definiciones, que coinciden en considerarlos como patrones consistentes en el tiempo que caracterizan a un grupo y dirigen su conducta, además de determinar y expresar la identidad tanto personal como grupal (Daniela et al, 2014; Närvänen, Kartastenpää y Kuusela, 2013; Pagalea y Uta, 2012).

Desde la teoría de la identidad social, el estilo de vida es un medio para formar la concepción y expresión que tiene cada persona acerca de sí misma y de su pertenencia o no a determinados grupos (Cătălin y Andreea, 2014). La identidad social proporciona a las personas normas, rituales y significados asociados a la forma en la que se debe vivir (Närvänen et al, 2013).

Uno de los campos en los que se ha abordado los estilos de vida es en el consumo. Los estudios de mercado buscan una comprensión de las personas y su comportamiento, con el fin de proporcionar a los vendedores de bienes y servicios, de los sectores público y privado, las herramientas para lograr sus objetivos de marketing. En este contexto, el interés por los estilos de vida cobra relevancia dado que el conocimiento de las variables sociodemográficas no eran suficientes para la segmentación adecuada del mercado (Veal, 1993). 
El estudio de los estilos de vida en el proceso de segmentación de mercados y la comprensión de los clientes objetivo se centra en la identificación de las actividades que la gente disfruta realizar, la delimitación de las temáticas de interés, sus opiniones y valores (Forehand, Deshpandé y Reed, 2002). Una de las formas de caracterizar estos elementos es a través de la compra, uso y descarte de productos y servicios, dado que como lo sostienen Grunert, Perrea, Zhou, Huang, Sørensen y Krystallis (2011), estos procesos no son sólo los medios de expresión de los estilos de vida, sino que, la gente busca congruencia entre el significado simbólico del producto y su propia forma de ver la vida (Forehand et al, 2002).

En este orden de ideas, el consumidor es, de manera holista, un agente social portador de una serie de estructuras semióticas; da sentido y cambia el valor de los objetos, así como el uso y adquisición de los mismos, razón por la cual, no puede ser abordado únicamente desde una visión reduccionista como un ser que procura solamente la satisfacción de sus necesidades básicas. De acuerdo con Baudrillard (1988), el consumo no tiene un enfoque exclusivamente utilitario, en el que los productos se limitan a responder a necesidades básicas, sino que se dan lugar a relaciones y códigos de significados, construidos social y culturalmente.

De esta forma, el consumo se ha convertido en un proceso de interacción social, en que los bienes y servicios son signos que informan sobre relaciones entre los miembros de un grupo y su distinción con otros grupos. En el espacio social los bienes y servicios adquieren valor por los significados más que por su uso (Baudrillard, 1988). Desde esta perspectiva, los bienes son medios de expresión de patrones de compra, estilos de vida y procesos psicológicos (Fenollar, 2003).

Según Chaves y Rodríguez-González (2013), existen diferentes tipos de consumo, los cuales se clasifican de acuerdo con el significado cultural que tiene para quien lo consume. Así, la tipología de consumos está circunscrita en: (a) consumo estatutario, (b) conspicuo, (c) hedónico, (d) identitario, (e) responsable, e (f) ideológico. Éstos se constituyen en áreas de interés para la comprensión del consumo como la construcción de relaciones sociales y símbolos, más que la satisfacción de necesidades primarias. En este contexto, la cultura y el consumo interactúan creando un sistema de significados (Fenollar y Ruiz, 2004) que constituye lo que se conoce como consumo simbólico.

Debido a las diferentes aproximaciones y definiciones de los estilos de vida y, por ser un constructo frecuentemente utilizado en Psicología del consumidor, el presente estudio tuvo por objetivo establecer el estado actual de la investigación en estilos de vida en esta área de aplicación mediante el desarrollo de una revisión sistemática de artículos científicos arbitrados por pares, publicados en los últimos 5 años en las bases de datos de referencia.

\section{Método}

\section{Tipo de estudio}

En este estudio se desarrolló un análisis de tipo descriptivo bibliométrico a partir de la revisión sistemática de literatura científica relacionada con el tema de los estilos de vida (Petticrew y Roberts, 2008). 


\section{Procedimiento}

La búsqueda de la información se llevó a cabo en agosto del 2015 en las bases de datos Science Direct ${ }^{\circledR}$, Springer Journal ${ }^{\circledR}$, Academic Search Complete ${ }^{\circledR}$ y Scopus ${ }^{\circledR}$. La combinación de las palabras consumer AND lifestyle fue usada en los campos de búsqueda, título, abstract, palabras clave. La revisión se realizó teniendo en cuenta un período de 6 años comprendidos entre 2010 y 2015. Se incluyeron artículos publicados y aquellos en versión pre-print.

Como parámetros para la depuración de la revisión se incluyeron solamente aquellos artículos que tuvieran la expresión lifestyle como variable de interés en los estudios; se excluyeron los artículos en los que lifestyle estaba relacionada con áreas de conocimiento diferentes a la psicología del consumidor (por ejemplo: psicología de la salud). Se diligenciaron los datos bibliográficos de los artículos que cumplieran con los criterios de inclusión en una matriz de Excel $^{\circledR}$, de la que posteriormente se efectuó la eliminación de los artículos duplicados.

Se diseñó una matriz en Excel ${ }^{\circledR} 2010$ para sistematizar la información de cada uno de los artículos, incluyendo los siguientes campos: base de datos de la que se descargó, revista de publicación, referencia en formato APA, abstract o resumen, tipo de consumo, definición de estilo de vida, palabras claves, autores, filiación institucional, año de publicación, continente de publicación, país donde se desarrolló el estudio. Además se incluyó el objetivo textual, metodología, tipo de estudio, diseño, tipo de artículo, unidad de análisis, tamaño muestral, características de la muestra y resultados. Se acudió a SCImago ${ }^{\circledR}$ para obtener el país de publicación. También fue necesaria la consulta de Google Scholar ${ }^{\circledR}$ para identificar el número de citas.

El número de artículos encontrados a partir de la búsqueda en las cuatro bases de datos fue de 816. No obstante, esta cantidad disminuyó a 94 artículos después de aplicar los criterios de exclusión y eliminar los artículos duplicados.

\section{Análisis de datos}

La base de datos obtenida se normalizó manualmente en Exce $^{\circledR} 2010$. Se procedió a realizar un análisis descriptivo de las variables de la base de datos y tablas dinámicas para visualizar las interacciones entre las variables de análisis.

De manera complementaria a los análisis bibliométricos, se realizó un análisis de contenido de las definiciones sobre estilos de vida. En primera instancia se desarrolló un análisis de convergencias de las definiciones según el tipo de consumo, posteriormente se establecieron las convergencias de las definiciones resultantes de cada tipo de consumo.

\section{Resultados}

En la figura 1 se presenta el número de artículos publicados en cada una de las bases de datos consultadas. La base de datos con más publicaciones fue Scopus ${ }^{\circledR}$, representando el 62\% del total de artículos, seguida por Science Direct® con 30\% de artículos publicados. En contraste, las publicaciones realizadas en Academic Search Complete ${ }^{\circledR}$ y Springer Journal ${ }^{\circledR}$ representan solo el 5\% y el 3\%, respectivamente. 


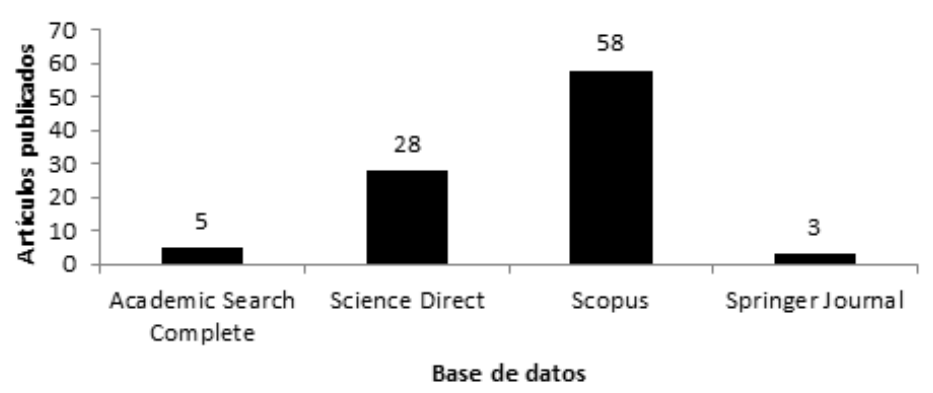

Figura 1. Número de artículos publicados en la temática por base de datos.

Un total de 346 palabras clave fueron identificadas en los artículos revisados. Como se muestra en la figura 2, el término más utilizado fue consumo sostenible, en 7 de los 94 artículos, seguido de la expresión comportamiento del consumidor usado en 6 artículos. Los términos análisis de conglomerados, segmentación de estilos de vida y materialismo, representan cada uno solo el $0,90 \%$ de las palabras clave utilizadas.

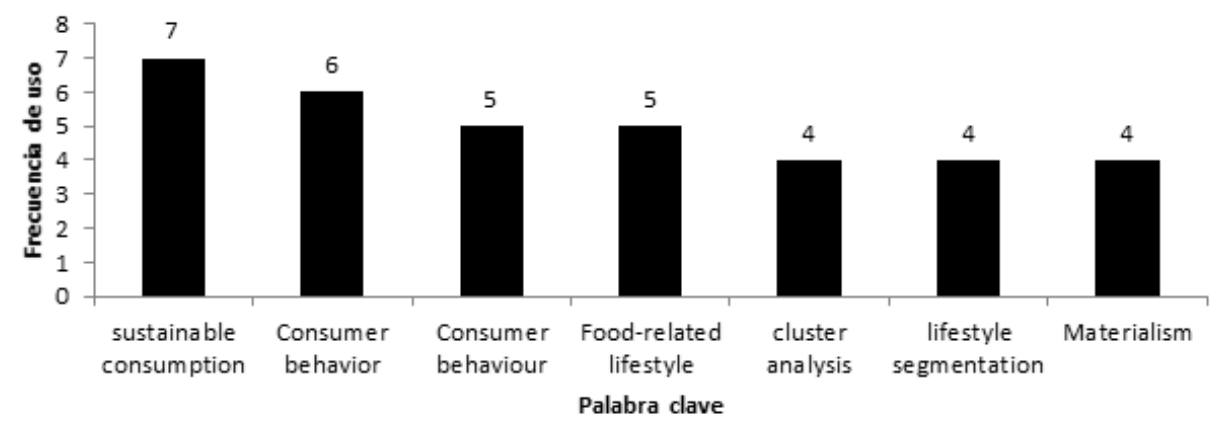

Figura 2. Frecuencias de palabras clave.

Las universidades que resultan ser generadoras de investigación en la temática de interés en esta revisión se pueden observar en la figura 3. De 153 instituciones solo 5 tienen mayor visibilidad frente al resto. Aarhus University representa el 4,1\% en contraste con RMIT University y Simon Fraser University que solo representan el $1,9 \%$ de las instituciones que promueven el desarrollo del conocimiento en el área de estilos de vida aplicados a consumidor.

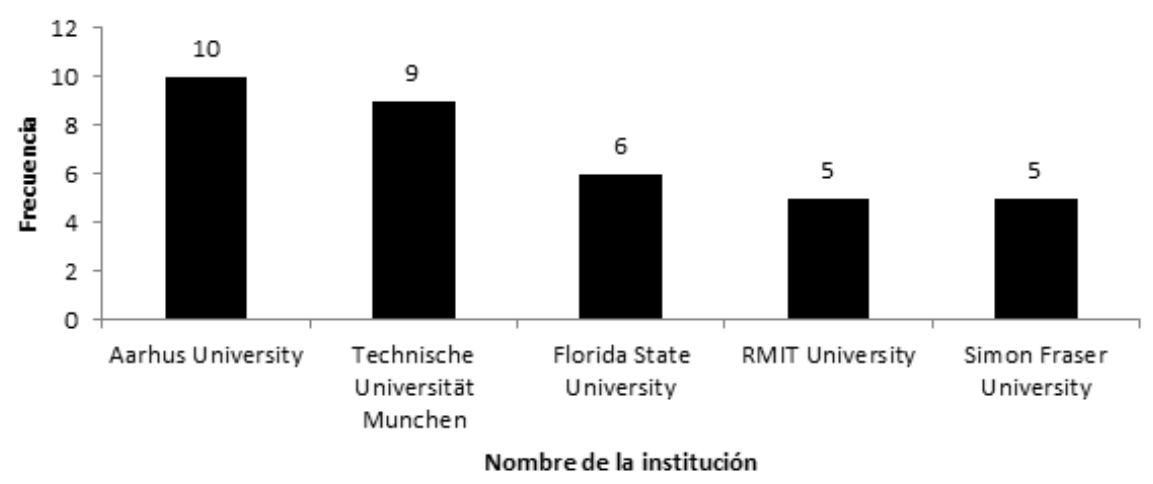

Figura 3. Filiación institucional. 
En la figura 4 se presenta el número de artículos relacionados con el año de publicación. El año 2012 fue el periodo con más publicaciones con un total de 24 artículos, seguido por el año 2011. En contraste, en el año 2010, solo hubo 6 publicaciones. Se puede evidenciar que posterior al año 2012 hubo un decremento en las publicaciones sobre el tema de interés para este estudio, sin embargo, es importante precisar que la búsqueda de información se llevó a cabo hasta agosto del 2015 por lo que el número de artículos de este último año no comprende todas las publicaciones del mismo.

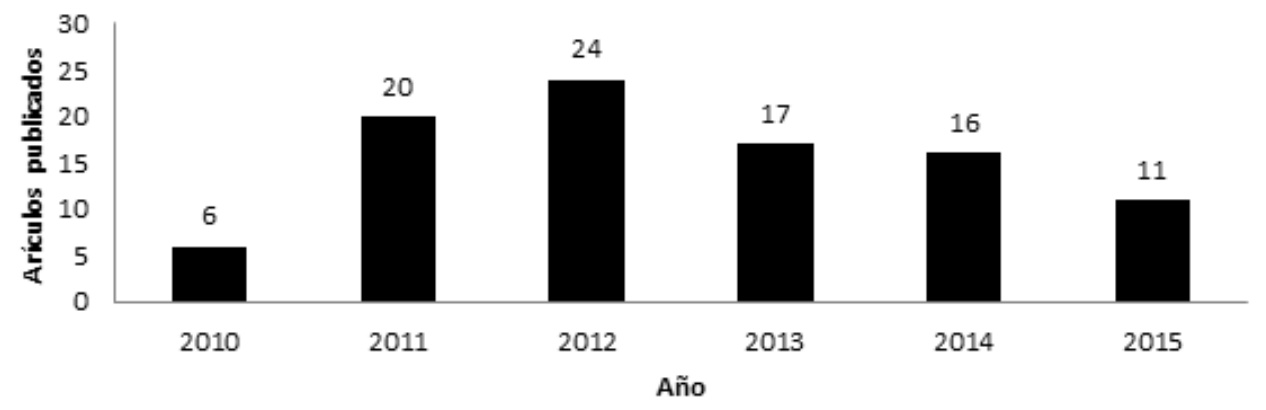

Figura 4. Número de artículos publicados por año entre 2010 y 2015.

Se realizó un análisis de las publicaciones respecto al país en el que se desarrolló el estudio. Como se evidencia en la figura 5, China es el país donde se desarrollaron la mayor cantidad de estudios, con 24 artículos, seguidos de Estados Unidos y Reino Unido. Australia y Brasil solo desarrollaron el 4\% de las investigaciones.

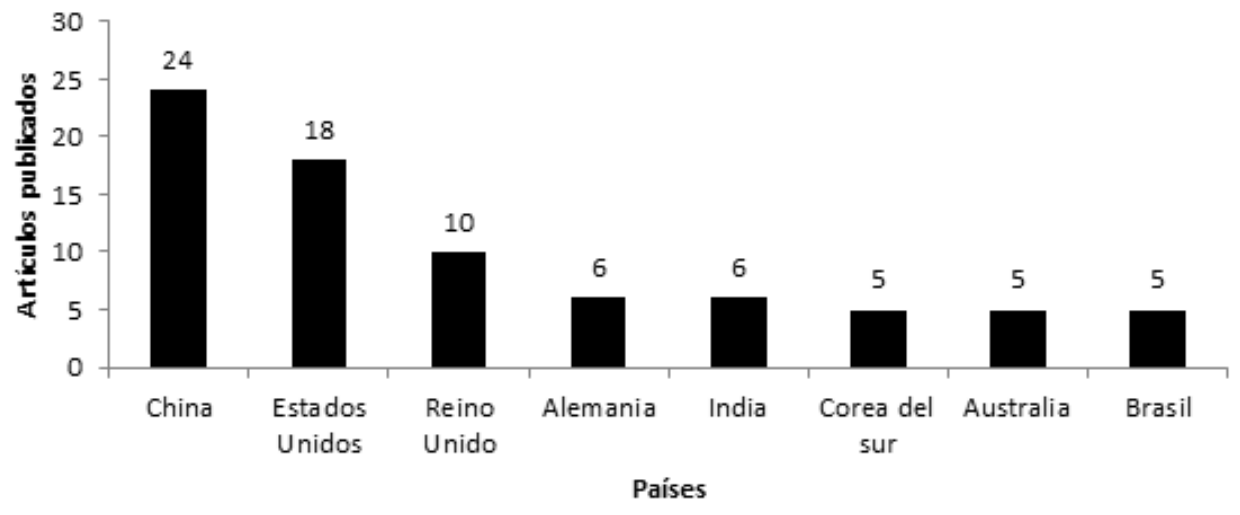

Figura 5. Países en los que se han desarrollado los estudios sobre Lifestyle.

El número de citas que había recibido cada artículo se obtuvo a través de Google Scholar ${ }^{\circledR}$, el rango de citas de los artículos tomados en esta revisión varía entre 0 y 97 . En la tabla 1 se presentan los artículos que han tenido 40 o más citaciones. El artículo de Chernev, Hamilton y Gal (2011), fue el más citado con 97 citas. 
Tabla 1

Número de citas por artículo

Referencia

Número

de citas

Chernev, A., Hamilton, R., \& Gal, D. (2011). Competing for Consumer Identity: Limits to SelfExpression and the Perils of Lifestyle Branding. Journal of Marketing, 75(3), 66-82. http:// doi.org/10.1509/jmkg.75.3.66

Nie, C., \& Zepeda, L. (2011). Lifestyle segmentation of US food shoppers to examine organic and local food consumption. Appetite, 57(1), 28-37. http://doi.org/10.1016/j.appet.2011.03.012

Jayasankara Prasad, C., \& Ramachandra Aryasri, A. (2011). Effect of shopper attributes on retail format choice behaviour for food and grocery retailing in India. International Journal of Retail \& Distribution Management, 39(1), 68-86. http://doi.org/10.1108/09590551111104486

Li, G., Li, G., \& Kambele, Z. (2012). Luxury fashion brand consumers in China: Perceived value, fashion lifestyle, and willingness to pay. Journal of Business Research, 65(10), 15161522. http://doi.org/10.1016/j.jbusres.2011.10.019

Jang, Y. J., Kim, W. G., \& Bonn, M. A. (2011). Generation Y consumers' selection attributes and behavioral intentions concerning green restaurants. International Journal of Hospitality Management, 30(4), 803-811. http://doi.org/10.1016/j.ijhm.2010.12.012

Hudders, L., \& Pandelaere, M. (2011). The Silver Lining of Materialism: The Impact of Luxury Consumption on Subjective Well-Being. Journal of Happiness Studies, 13(3), 411-437. http:// doi.org/10.1007/s10902-011-9271-9

Lee, E. M., Park, S.-Y., Rapert, M. I., \& Newman, C. L. (2012). Does perceived consumer fit matter in corporate social responsibility issues? Journal of Business Research, 65(11), 15581564. http://doi.org/10.1016/j.jbusres.2011.02.040

Alebaki, M., \& Iakovidou, O. (2011). Market segmentation in wine tourism: A comparison of approaches. Tourismos, 6(1), 123-140. Retrieved from http://www.scopus.com/inward/record. url?eid=2-s2.0-79953291250\&partnerID=tZOtx3y1

Michaelidou, N., \& Hassan, L. M. (2010). Modeling the factors affecting rural consumers' purchase of organic and free-range produce: A case study of consumers' from the Island of Arran in Scotland, UK. Food Policy, 35(2), 130-139. http://doi.org/10.1016/j.foodpol.2009.10.001

La investigación llevada a cabo en éste artículo se basa en la hipótesis de que los consumidores utilizan las marcas para expresar su identidad, lo que ha llevado a muchas empresas a reposicionar sus productos, centrándose en los atributos funcionales y en cómo encajan estos en el estilo de vida del consumidor. Los resultados indican que la necesidad de autoexpresión y la preferencia por determinadas marcas dependen de la disponibilidad de medios alternativos de autoexpresión. Así, por ejemplo, comportamientos auto-expresivos como la personalización de los productos, pueden llevar a generar identidad en el consumidor.

Por otro lado, respecto a los tipos de metodología y de estudio, se identificaron 63 artículos con una orientación cuantitativa, constituyendo el $67 \%$ del total de artículos (ver figura 6). Se evidenció que la mayoría de los artículos analizados presentaban estudios de correlacionales (35,1\%), seguido de los descriptivos con un $21,3 \%$, constituyendo el 56,4\% del total de artículos. En contraste, los estudios de tipo explicativo, instrumental y exploratorio representan tan solo el 7,4\%, $2,1 \%$ y $1,1 \%$ respectivamente (ver tabla 2). 
Tabla 2

Tipo de estudio en estilos de vida

\begin{tabular}{cc}
\hline Tipo de estudio & Frecuencia \\
\hline Correlacional & 33 \\
Descriptivo & 20 \\
Hermenéutico & 15 \\
Mixto & 10 \\
Explicativo & 7 \\
Etnográfico & 5 \\
Exploratorio & 2 \\
Fenomenológico & 1 \\
Instrumental & 1 \\
\hline
\end{tabular}

Veintiún estudios se desarrollaron con una metodología de corte cualitativo, los cuales representan el $22 \%$ del total de artículos (ver figura 6). Los estudios hermenéuticos constituyeron el $16 \%$ de los artículos. Por su parte, los estudios etnográficos y fenomenológicos representan solo el $5 \%$ y el $1 \%$ de los métodos utilizados para abordar los estilos de vida (ver tabla 2). Finalmente, la metodología mixta representa el 11\% (10 artículos) del total de artículos analizados (ver figura 6).

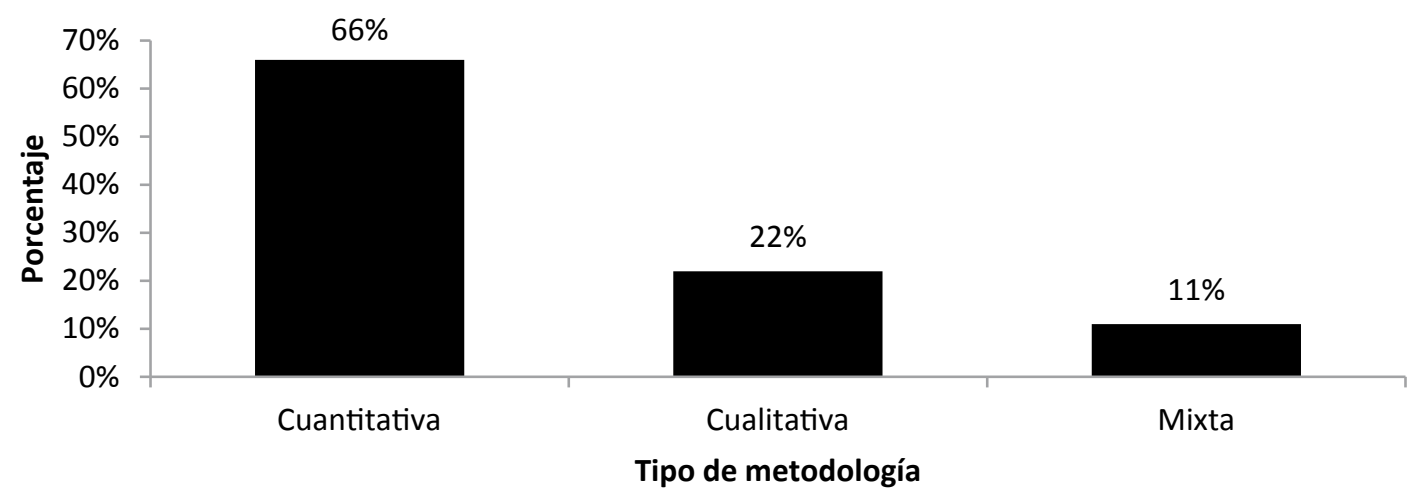

Figura 6. Porcentajes tipo de metodología en los artículos sobre lifestyle.

Por otro lado, en la tabla 3, se presentan los datos correspondientes a la variable tipo de diseño. Allí se muestra que el tipo de diseño más utilizado fue el transversal (62\% de los artículos), en contraste con los diseños experimentales que solo representan el $7 \%$ de los datos. Uno de los artículos fue de tipo instrumental por lo que no aplica algún tipo de diseño. 
Tabla 3

Tipo de diseño en estilos de vida

\begin{tabular}{cc}
\hline Tipo de diseño & Frecuencia \\
\hline Transerval & 58 \\
Emergente & 18 \\
Mixto & 10 \\
Experimental & 7 \\
N/A & 1 \\
\hline
\end{tabular}

En cuanto a la variable tipo de consumo, como se evidenció en la tabla 4, se encontró que de los 94 trabajos revisados 30 abordaban el consumo responsable, seguido por el consumo hedónico (18 artículos) y el consumo identitario (17). Los trabajos referidos al consumo ideológico (8 artículos), utilitario (8 artículos), estatutario (7) y conspicuo (5) tienen una frecuencia más baja.

Tabla 4

Número de artículos por tipo de consumo

\begin{tabular}{lc}
\hline Tipo de consumo & Frecuencia \\
\hline Consumo responsable & 30 \\
Consumo hedónico & 18 \\
Consumo identitario & 17 \\
Consumo ideológico & 8 \\
consumo utilitario & 8 \\
Consumo estatutario & 7 \\
Consumo conspicuo & 5 \\
Consumo hedónico. Consumo utilitario. & 1 \\
\hline
\end{tabular}

De manera complementaria a los análisis bibliométricos se elaboró un análisis de contenido de las definiciones sobre estilos de vida; considerando que únicamente 71 de los 94 artículos tenían definida esta variable. En la tabla 5, se pueden evidenciar las definiciones por tipo de consumo, resultado de las convergencias que se identificaron en las definiciones dadas en los artículos. Se trabajó con 7 tipos de consumo, consumo conspicuo, estatutario, hedónico, identitario, ideológico, responsable y utilitario. 
Tabla 5

Definiciones de estilo de vida de acuerdo al tipo de consumo

\begin{tabular}{ll}
\hline Categoría & \multicolumn{1}{c}{ Definición estilos de vida } \\
\hline & $\begin{array}{l}\text { En el consumo conspicuo los estilos de vida son entendidos como comportamientos observables } \\
\text { que reflejan necesidades internas y deseos del consumidor. Representan las características } \\
\text { distintivas describen a un grupo y lo distinguen de los demás. Son reacciones o respuesta } \\
\text { a estímulos culturales, individuales y sociales. Permite predecir el comportamiento del }\end{array}$ \\
consumidor, es un medio para entender la imagen idealizada del consumidor y de cómo quiere \\
conspicuo & $\begin{array}{l}\text { que otros lo vean, es decir, que es la forma en que se clasifican en términos individuales y } \\
\text { grupales sobre la base de las cosas que hacen y cómo eligen invertir su dinero. Las actividades, } \\
\text { los intereses, las opiniones y la elección de productos o servicios son indicadores del estilo del } \\
\text { consumidor. } \\
\text { Los principales métodos usados para su medición son AIO (actividades, intereses y opiniones), } \\
\text { VALS (estilos de vida y valores) y LOV (valores). }\end{array}$ \\
\hline
\end{tabular}

En consumo estatutario los estilos de vida se concentran en aquellos comportamientos distintivos de vida cuyos determinantes pueden ser de carácter social, individual o cultural; estos elementos únicos describen a un grupo y lo distinguen de los demás, ya que dentro de estos se mantienen unas reglas, rituales y significados de consumo. Se constituye de la

Consumo estatutario interacción con el ambiente u otros individuos. Los intereses, las opiniones y la elección de productos o servicios son indicadores del estilo del consumidor. Tiene como característica la consistencia, la cual permite predecir el comportamiento futuro.

Por lo que, el estilo de vida de lujo puede ser descrito en términos de la actitud personal hacia un producto de marca particular que trae la satisfacción de las necesidades psicológicas y funcionales del individuo, es una expresión material de la propia identidad.

Los estilos de vida, desde el consumo hedónico, son entendidos como un espacio social en el que hay patrones consistentes de consumo y en el que la cercanía entre consumidores con respecto a variables culturales, sociales y psicológicas da lugar a la caracterización de un grupo social y la diferenciación de éste con respecto a otros grupos; con lleva a un modo distintivo de vida. Se constituye de la interacción con el ambiente $u$ otros individuos. A partir del consumo se representa la identidad del consumidor, incluyendo los productos que uno compra, cómo los utiliza, cómo se piensa de ellos y cómo se siente acerca de ellos. Las actividades, los intereses, las opiniones y la elección de productos o servicios son indicadores del estilo del consumidor.

Consumo

hedónico Por lo tanto, el estilo de vida es cómo se vive, es la imagen que tiene el consumidor sí mismo y las metas o motivaciones del consumidor, la cual es resultado de cómo fue socializado en su cultura, mediante la creación de símbolos culturales que reflejan la identidad del consumidor. Tiene en cuenta el lugar donde se lleva a cabo el consumo y la búsqueda recompensas intrínsecas como placer, diversión, aventura y distracción a través de los productos. Los principales métodos usados para su medición son AIO, VALS (estilos de vida y valores), LOV y teoría impulsada de espacio social.

Desde el consumo identitario los estilos de vida son entendidos como una forma o medio de expresión, en la que algunos productos se convierten en objetos de representación que satisfacen una necesidad de autoidentificación y autodefinición compartida en términos de las propiedades que definen a un grupo particular. Se reflejan en patrones de acción consistentes. Los estilos de vida de los consumidores vinculan su identidad del yo y social, la que se refleja en el consumo de productos y marcas. Así pues, el consumidor busca congruencia entre el significado simbólico del producto y sus propias formas de ver la vida en general.

Consumo identitario Los estilos de vida permiten predecir y son el conjunto de comportamientos de los consumidores
(cómo los consumidores piensan, viven, actúan, sienten) que reflejan variables culturales, sociológicas y psicológicas. Además, permiten diferenciar a los grupos de personas, entendiendo lo que hace la gente y por qué lo hacen. Son resultado del moldeamiento e interacción social, proporcionando signos cargados de significados culturales, normas, funciones y criterios para vivir. Han sido evaluados a través de los métodos de AIO, VALS. 
Los estilos de vida desde el consumo ideológico hacen referencia a la orientación que un individuo y su grupo desarrollan y siguen al apropiar o difundir creencias que median en el consumo de productos o servicios, dichas creencias están basadas en el contexto cultural y medio psicosocial relacionado con la etapa de vida de los individuos. Son conductualmente observables y dan regularidad a las prácticas de consumo similares que adopta el grupo.

Consumo Son una construcción colectiva y simbólica que se construye como referencia a otros grupos ideológico específicos, que permite agrupar a los consumidores en grupos en los que las personas se comportan de manera similar. Describe los roles que las personas desempeñan en la vida y cómo ellos piensan que deberían cumplir dichos roles. Por lo tanto, los estilos de vida deben incluir su relación con los cambios en las estructuras sociales, culturales y elementos psicológicos.

Los estilos de vida en el consumo responsable se definen como elementos cognitivos y conativos de los consumidores asociados con los productos y servicios que se compran, usan y desechan; dicho grupo de actividades, intereses y opiniones reflejan patrones consistentes que las personas siguen en sus vidas. Los estilos de vida reafirman y moderan la identidad del consumidor, social y cultural, representando la manera en que las personas desean vivir. Se relacionan con aquellos elementos únicos que describen a un grupo y lo distingue de otros, por lo que se considera un criterio de clasificación social. Es resultado de las dimensiones

Consumo

responsable Se entienden como la disposición a participar en comportamientos de consumo (selección simbólicas producto de las interacciones con el ambiente. de productos, uso y desecho de los mismos) que impliquen temas sociales, ambientales, alimentación saludable y con los animales, de acuerdo con los valores personales del consumidor, de esta manera se adapta el comportamiento del consumidor a conseguir los mismos valores básicos en todas las facetas de su vida. Por lo tanto, el acto de consumo que caracteriza los estilos de vida involucra aspectos de responsabilidad social, información específica sobre los bienes y servicios y del contexto socio-económico y cultural. Tiene en cuenta variables sociales, culturales y psicológicas. Métodos utilizados para medir el estilo de vida AIO y VALS.

Desde el consumo utilitario los estilos de vida se conciben como la forma en la que vive un individuo, en la que lo que prima es la utilidad u objetivo de aquello que se consume, así como los atributos del producto, que satisfacen y guían las necesidades básicas del consumidor. Supone la mediación entre percepciones y comportamientos específicos del producto. Se define por las características de un determinado grupo, que permiten a su vez, diferenciarlo de

Consumo utilitario otros grupos. Se ve afectado no solo por dimensiones psicológicas del consumidor sino por dimensiones socio-culturales (cultura, grupos de referencia, la familia, clase social), lo cual influye en la elección de productos y marcas.

Son comportamientos consistentes, influenciados y modelados por un grupo social de referencia, orientados a los productos, eventos o recursos. Por lo que, los intereses y necesidades son susceptibles de ser compartidas.

De acuerdo con el análisis de contenido realizado, se pueden definir los estilos de vida en el área de consumidor como una forma distintiva y consistente de vida, en la que están implicados procesos psicológicos, sociales y culturales. Estos procesos median en el proceso de compra, uso y descarte de bienes y servicios.

\section{Discusión}

El propósito de este estudio fue hacer una revisión de los artículos publicados en revistas científicas sobre los estilos de vida en el área de consumidor entre los años 2010 y 2015 . El estudio permitió conocer la producción científica en la temática de intereses, identificando la representación de las fuentes más citadas, las instituciones que promueven la producción en este tema, los países en los que se desarrollan los estudios, la metodología utilizada y el tipo de método que contribuye a sistematizar la investigación y favorece el logro de los objetivos establecidos. 
Uno de los aspectos importantes a resaltar está relacionado con las palabras clave. Las palabras clave representan una herramienta fundamental a la hora de realizar búsquedas bibliográficas, proporcionando acceso a todos los trabajos relacionados con la temática; consolidados en las bases de datos científicas. En los resultados de este documento se encontró que únicamente 7 palabras tienen uso relativamente frecuente, lo que implica que 337 tienen un uso de máximo 3 veces. Esto puede dificultar la difusión de los documentos en esta área e incluso tener problemas al momento de su identificación (Granda Orive, García Río y Callol Sánchez, 2003; González Tous, y Mattar, 2012).

Tal dispersión podría estar asociada con la poca precisión en las definiciones dadas en los artículos sobre los estilos de vida; aunque los artículos de esta revisión tenían por objetivo estudiar los estilos de vida, partieron de identificadores difusos, dejando de lado información relevante para la formulación de referentes teóricos consistentes y precisos. Como lo mencionan González Tous y Mattar (2012), las palabras clave no son sólo útiles para las búsquedas bibliográficas sino que pueden servir para analizar y clasificar las producciones científicas por temáticas de intereses y corrientes de indagación para el investigador.

En efecto, debido a la gran cantidad de definiciones, la poca precisión en las mismas y la gran diversidad en los usos, fue necesario replantearse su definición y las dimensiones que la constituyen, de acuerdo a las temáticas de interés (tipos de consumo) de la presente investigación.

De acuerdo con el análisis de contenido, se pueden definir los estilos de vida en el área de consumidor como una forma distintiva y consistente de vida, en la que están implicados procesos psicológicos, sociales y culturales, que median en el proceso de compra, uso y descarte de bienes y servicios. En este sentido, teniendo en cuenta los postulados de Chaves y Rodríguez-González (2013), el consumidor no puede entenderse solo con los procesos psicológicos (motivaciones, actitudes, necesidades) sino que deben incluir la cultura (productos como signos y significados para expresar la realidad social) y procesos de socialización (formas de intercambio e identificación del su rol social).

Como se pudo evidenciar, la relación con un objeto que ha adquirido una propiedad de símbolo, puede caracterizar estilos de vida, identidades de grupo y/o prácticas de consumo (Baudrillard, 1998; Fenollar y Ruiz, 2004; Tajfel, 1984). Los bienes y servicios se orientan a la satisfacción de necesidades ajenas a su función primaria, ya que se personalizan y generalizan en la cultura y contexto en la que estén inmersos los consumidores (Barreto y Neme, 2014; Neme, 2013).

De acuerdo con lo anterior, los estilos de vida y las prácticas de consumo dependen del contexto social y cultural en el que se da la interacción del grupo social (Neme, 2013). Debido a que en las últimas décadas las interacciones sociales no sólo tienen lugar en espacios físicos sino a través de internet, es importante que futuras investigaciones sobre estilos de vida en el área de consumidor se realicen teniendo en cuenta nuevos contextos de interacción, como por ejemplo las redes sociales digitales, en las que es posible la construcción de estilos de vida, identidades de grupo y/o prácticas de consumo. 
Los espacios virtuales constituyen una forma de participación que, como ha señalado Castells (2012), ofrece posibilidades de interacción en la que los consumidores forman sus propias concepciones políticas e ideológicas. Se han convertido en plataformas para todo tipo de actividad, no solo personal sino para el marketing, el comercio electrónico, la educación, la distribución de los medios de comunicación, entre otros escenarios de aplicación.

En consecuencia, el desarrollo de estudios bibliométricos en esta área de interés permitiría orientar la formulación de investigaciones consistentes con los nuevos escenarios de interacción como las redes sociales digitales, a través de la formulación de definiciones consistentes y modelos integradores, que sustenten las dinámicas de desarrollo de la producción de conocimiento.

\section{Referencias}

Ahmad, R. (2012). Habitus, Capital, and Patterns of Taste in Tourism Consumption: A Study of Western Tourism Consumers in India. Journal of Hospitality \& Tourism Research, 38(4), 487-505. http://doi.org/10.1177/1096348012461550

Alebaki, M., \& Iakovidou, O. (2011). Market segmentation in wine tourism: A comparison of approaches. Tourismos, 6(1), 123-140. Recuperado de: http://www.scopus. com/inward/record.url?eid=2-s2.0-79953291250\&partnerID=tZOtx3y1

Axsen, J., Bailey, J., \& Castro, M. A. (2015). Preference and lifestyle heterogeneity among potential plug-in electric vehicle buyers. Energy Economics, 50, 190-201. http://doi.org/10.1016/j.eneco.2015.05.003

Axsen, J., Orlebar, C., \& Skippon, S. (2013). Social influence and consumer preference formation for pro-environmental technology: The case of a U.K. workplace electric-vehicle study. Ecological Economics, 95, 96-107. http://doi.org/10.1016/j. ecolecon.2013.08.009

Axsen, J., TyreeHageman, J., \& Lentz, A. (2012). Lifestyle practices and pro-environmental technology. Ecological Economics, 82, 64-74. http://doi.org/10.1016/j.ecolecon.2012.07.013

Barber, N., \& Taylor, C. (2011). Equity benefits of smaller wine regions and lifestyle segmentation. Journal of Brand Management, 19(2), 158-175. http://doi. org/10.1057/bm.2011.18

Barlés-Arizón, M. J., Fraj-Andrés, E., \& Martínez-Salinas, E. (2013). Family Vacation Decision Making: The Role of Woman. Journal of Travel \& Tourism Marketing, 30(8), 873-890. http://doi.org/10.1080/10548408.2013.835681

Barreto, I., \& Neme, S. R. (2014). Eficacia de tácticas de influencia en la intención de conducta proambiental. Revista Latinoamericana de Psicología, 46(2), 111-116.

Baudrillard, J. (1988). El sistema de los objetos. México: Siglo xxi editores.

Bernués, A., Ripoll, G., \& Panea, B. (2012). Consumer segmentation based on convenience orientation and attitudes towards quality attributes of lamb meat. Food Quality and Preference, 26(2), 211-220. http://doi.org/10.1016/j.foodqual.2012.04.008

Blasius, J., \& Mühlichen, A. (2010). Identifying audience segments applying the "social space" approach. Poetics, 38(1), 69-89. http://doi.org/10.1016/j.poetic.2009.10.003

Bond, S. (2011). Barriers and drivers to green buildings in Australia and New Zealand. Journal of Property Investment \& Finance, 29(4/5), 494-509. http://doi. org/10.1108/14635781111150367 
Bouvier, G. (2012). How Facebook users select identity categories for self-presentation. Journal of Multicultural Discourses, 7(1), 37-57. http://doi.org/10.1080/17447143. 2011.652781

Castells, M. (2012). Redes de indignación y esperanza: los movimientos sociales en la era de internet. Madrid: Alianza Editorial.

Cătălin, M. C., \& Andreea, P. (2014). Brands as a Mean of Consumer Self-expression and Desired Personal Lifestyle. Procedia - Social and Behavioral Sciences, 109, 103-107. http://doi.org/10.1016/j.sbspro.2013.12.427

Chan, K. S. (2010). Traditionality and Hybridity: A Village Cuisine in Metropolitan Hong Kong. Visual Anthropology, 24(1-2), 171-188. http://doi.org/10.1080/089494 68.2011.527818

Chang, C.-C., Tsai, J.-M., Hung, S.-W., \& Lin, B.-C. (2015). A hybrid decision-making model for factors influencing the purchase intentions of technology products: the moderating effect of lifestyle. Behaviour \& Information Technology, 1-15. http:// doi.org/10.1080/0144929X.2015.1019566

Chaves, S., \& Rodríguez-González, L. (2013). Consumo simbólico: una perspectiva sociocultural en la comprensión del comportamiento del consumidor. Revista Iberoamericana de Psicología: Ciencia y Tecnología, 6(2), 27-34.

Chen, J., \& Lobo, A. (2012). Organic food products in China: determinants of consumers' purchase intentions. The International Review of Retail, Distribution and Consumer Research, 22(3), 293-314. http://doi.org/10.1080/09593969.2012.68259 6

Chiu, W., Kim, H. H., Lee, Y. A., \& Won, D. (2014). Application of a modified internet shopper lifestyle scale to Taiwanese college-age sporting goods consumers. Social Behavior and Personality, 42(8), 1245-1256. http://doi.org/10.2224sbp.2014.42.8.1245

Chernev, A., Hamilton, R., \& Gal, D. (2011). Competing for Consumer Identity: Limits to Self-Expression and the Perils of Lifestyle Branding. Journal of Marketing, 75(3), 66-82. http://doi.org/10.1509/jmkg.75.3.66

Daniela, R. R., Roxana, S., \& Elena, C. (2014). Romanian Consumer Attitude towards Precious Metals Products Purchase. Procedia - Social and Behavioral Sciences, 109, 350-354. http://doi.org/10.1016/j.sbspro.2013.12.470

Dansiricha, R., \& Suwunnamek, O. (2014). A Comparison of Thai Consumers Purchasing Behaviour with the Environmental Characteristics: Electric Appliances Market. Research Journal of Business Management, 8(4), 338-352. http://doi. org/10.3923/rjbm.2014.338.352

Fenollar, P. (2003). Estilos de vida: Paradigmas del mercado. (Tesis doctoral). Universidad complutense de Madrid. Madrid. Recuperado de: http://www.ucm.es/BUCM/ tesis/cps/ucm-t27084.pdf

Fenollar, P., \& Ruiz, S. (2004). Efectos de los medios de comunicación, la imagen social de la publicidad y los otros en el consumo simbólico. Universidad de Murcia, 1-8. Recuperado de: http://www.epum2004.ua.es/aceptados/258.pdf.

Forehand, M., Deshpandé, R., \& Reed, A. (2002). Identity salience and the influence of differential activation of the social self-schema on advertising response. Journal of Applied Psychology, 87(6), 1086-1099 
Ganglmair-Wooliscroft, A., \& Lawson, R. (2010). Subjective Well-Being of Different Consumer Lifestyle Segments. Journal of Macromarketing, 31(2), 172-183. http:// doi.org/10.1177/0276146710393251

Gao, S., Chen, Z., Zheng, W., \& Zhou, W. (2012). An exploratory study on lifestyles and the adoption of mobile services in China. In Proceedings of the 10th International Conference on Advances in Mobile Computing \& Multimedia - MoMM '12 (p. 249). New York, New York, USA: ACM Press. http://doi.org/10.1145/2428955.2429002

Goldsmith, R. E., Reinecke Flynn, L., \& Clark, R. A. (2014). The etiology of the frugal consumer. Journal of Retailing and Consumer Services, 21(2), 175-184. http://doi. org/10.1016/j.jretconser.2013.11.005

González Tous, M., \& Mattar, S. (2012). Las claves de las palabras clave en los artículos científicos. Revista MVZ Córdoba, 17(2), 2955-2956.

Gordon, R., Gurrieri, L., \& Chapman, M. (2015). Broadening an understanding of problem gambling: The lifestyle consumption community of sports betting. Journal of Business Research. http://doi.org/10.1016/j.jbusres.2015.03.016

Granda Orive, J., García Río, F., \& Callol Sánchez, L. (2003). Importancia de las palabras clave en las búsquedas bibliográficas. Revista española de salud pública, 77(6), 765-767. Recuperado el 11 de noviembre del 2015 de http://www.scielosp. org/scielo.php?script=sci_arttext\&pid=S1135-57272003000600010\&lng=en\&tlng =es.

Grinstein, A., \& Wathieu, L. (2012). Happily (mal)adjusted: Cosmopolitan identity and expatriate adjustment. International Journal of Research in Marketing, 29(4), 337-345. http://doi.org/10.1016/j.ijresmar.2012.03.003

Grunert, K. G., Perrea, T., Zhou, Y., Huang, G., Sørensen, B. T., \& Krystallis, A. (2011). Is food-related lifestyle (FRL) able to reveal food consumption patterns in non-Western cultural environments? Its adaptation and application in urban China. Appetite, 56(2), 357-67. http://doi.org/10.1016/j.appet.2010.12.020

Hasnah Hassan, S., Ramayah, T., Mohamed, O., \& Maghsoudi, A. (2015). E-lifestyle, Customer Satisfaction, and Loyalty among the Generation Y Mobile Users. Asian Social Science, 11(4), 157-168. http://doi.org/10.5539/ass.v11n4p157

He, Y., Zou, D., \& Jin, L. (2010). Exploiting the goldmine: a lifestyle analysis of affluent Chinese consumers. Journal of Consumer Marketing, 27(7), 615-628. http://doi. org/10.1108/07363761011086362

Henningsen, L. (2012). Individualism for the masses? Coffee consumption and the Chinese middle class' search for authenticity. Inter-Asia Cultural Studies, 13(3), 408-427. http://doi.org/10.1080/14649373.2012.689709

Hoque, N. (2013). Analysing Sustainable Consumption Patterns: A literature review. Development, 56(3), 370-377. http://doi.org/10.1057/dev.2014.13

Huang, X. (2012). Lifestyles in virtual communities: collaborative consumption and interaction. Chinese Journal of Communication, 5(1), 109-127. http://doi.org/10.10 80/17544750.2011.640542

Hudders, L., \& Pandelaere, M. (2011). The Silver Lining of Materialism: The Impact of Luxury Consumption on Subjective Well-Being. Journal of Happiness Studies, 13(3), 411-437. http://doi.org/10.1007/s10902-011-9271-9 
Husić-Mehmedović, M., Čičić, M., \& Agić, E. (2015). Regional Lifestyle Segmentation in the Western Balkans. South East European Journal of Economics and Business, 9(2), 46-53. http://doi.org/10.2478/jeb-2014-0007

Jafari, A., \& Goulding, C. (2013). Globalization, reflexivity, and the project of the self: a virtual intercultural learning process. Consumption Markets \& Culture, 16(1), 65-90. http://doi.org/10.1080/10253866.2012.659435

Jang, Y. J., Kim, W. G., \& Bonn, M. A. (2011). Generation Y consumers' selection attributes and behavioral intentions concerning green restaurants. International Journal of Hospitality Management, 30(4), 803-811. http://doi.org/10.1016/j. ijhm.2010.12.012

Jang, Y. J., Kim, W. G., \& Yang, I.-S. (2011). Mature consumers' patronage motives and the importance of attributes regarding HMR based on the food-related lifestyles of the upper middle class. International Journal of Hospitality Management, 30(1), 55-63. http://doi.org/10.1016/j.ijhm.2010.06.001

Jayasankara Prasad, C., \& Ramachandra Aryasri, A. (2011). Effect of shopper attributes on retail format choice behaviour for food and grocery retailing in India. International Journal of Retail \& Distribution Management, 39(1), 68-86. http:// doi.org/10.1108/09590551111104486

Kavak, B., Tektaş, Ö. Ö., Eryi $\square$ it, C., \& Başgöze, P. (2010). Effects of hedonic-utilitarian motives and life style on attitudinal-behavioral loyalty. Bogazici Journal, 24(1-2), 21-40. Retrieved from http://www.scopus.com/inward/record.url?eid=2s2.0-79954621607\&partnerID=tZOtx3y1

Kim, D., \& (Shawn) Jang, S. (2014). Motivational drivers for status consumption: A study of Generation Y consumers. International Journal of Hospitality Management, 38, 39-47. http://doi.org/10.1016/j.ijhm.2013.12.003

Ko, E., Taylor, C. R., Sung, H., Lee, J., Wagner, U., Navarro, D. M.-C., \& Wang, F. (2012). Global marketing segmentation usefulness in the sportswear industry. Journal of Business Research, 65(11), 1565-1575. http://doi.org/10.1016/j.jbusres.2011.02.041

Korjenic, A., \& Bednar, T. (2011). Impact of lifestyle on the energy demand of a single family house. Building Simulation, 4(2), 89-95. http://doi.org/10.1007/s12273-0100013-4

Krishnan, J. (2011). Lifestyle - A tool for understanding buyer behavior. International Journal of Economics and Management, 5(2), 283-298. Retrieved from http://www. scopus.com/inward/record.url?eid=2-s2.0-84869453866\&partnerID=tZOtx3y1

Kucukusta, D., \& Denizci Guillet, B. (2015). Lifestyle Segmentation of Spa Users: A Study of Inbound Travelers to Hong Kong. Asia Pacific Journal of Tourism Research, 1-20. http://doi.org/10.1080/10941665.2015.1025087

Lee, E. M., Park, S.-Y., Rapert, M. I., \& Newman, C. L. (2012). Does perceived consumer fit matter in corporate social responsibility issues? Journal of Business Research, 65(11), 1558-1564. http://doi.org/10.1016/j.jbusres.2011.02.040

Lewis, T. (2012). "There grows the neighbourhood": Green citizenship, creativity and life politics on eco-TV. International Journal of Cultural Studies, 15(3), 315-326. http://doi.org/10.1177/1367877911433753

Lewis, T., Martin, F., \& Sun, W. (2012). Lifestyling Asia? Shaping modernity and selfhood on life-advice programming. International Journal of Cultural Studies, 15(6), 537-566. http://doi.org/10.1177/1367877912451693 
Li, G., Li, G., \& Kambele, Z. (2012). Luxury fashion brand consumers in China: Perceived value, fashion lifestyle, and willingness to pay. Journal of Business Research, 65(10), 1516-1522. http://doi.org/10.1016/j.jbusres.2011.10.019

Liang, A. R.-D., \& Lim, W. M. (2011). Exploring the online buying behavior of specialty food shoppers. International Journal of Hospitality Management, 30(4), 855-865. http://doi.org/10.1016/j.ijhm.2011.01.006

Lin, C.-S., \& Chou, H.-Y. (2013). Dimensions and Effects of Consumers' Travel-Transportation Involvement: The Case of the Mini Three Links. Asia Pacific Journal of Tourism Research, 20(1), 51-75. http://doi.org/10.1080/10941665.2013.866584

Lin, F.-Y. (2013). Exploring Alternative Lifestyle Segmentation Schemes for Travel Volunteers. Asia Pacific Journal of Tourism Research, 19(4), 416-427. http://doi.or $\mathrm{g} / 10.1080 / 10941665.2013 .764334$

Lin, E.-Y. (2012). Starbucks as the Third Place: Glimpses into Taiwan's Consumer Culture and Lifestyles. Journal of International Consumer Marketing, 24(1-2), 119-128. http://doi.org/10.1080/08961530.2012.650142

Luo, L., Ratchford, B. T., \& Yang, B. (2013). Why We Do What We Do: A Model of Activity Consumption. Journal of Marketing Research, 50(1), 24-43. http://doi. org/10.1509/jmr.10.0416

McDonald, S., Oates, C. J., Alevizou, P. J., Young, C. W., \& Hwang, K. (2012). Individual strategies for sustainable consumption. Journal of Marketing Management, 28(3-4), 445-468. http://doi.org/10.1080/0267257X.2012.658839

Michaelidou, N., \& Hassan, L. M. (2010). Modeling the factors affecting rural consumers' purchase of organic and free-range produce: A case study of consumers' from the Island of Arran in Scotland, UK. Food Policy, 35(2), 130-139. http://doi. org/10.1016/j.foodpol.2009.10.001

Mishra, A., Dash, S. B., \& Cyr, D. (2014). Linking user experience and consumer-based brand equity: the moderating role of consumer expertise and lifestyle. Journal of Product \& Brand Management, 23(4/5), 333-348. http://doi.org/10.1108/JPBM12-2013-0459

Mohamed Saat, M. K., Sedon, M. F., \& Dom, M. (2014). Re-Interpretingurbanluxury culture through consumerism. World Applied Sciences Journal, 30(30 A), 70-80. http://doi.org/10.5829/idosi.wasj.2014.30.icmrp.11

Montoya, D. Y., \& Scott, M. L. (2013). The Effect of Lifestyle-Based Depletion on Teen Consumer Behavior. Journal of Public Policy \& Marketing, 32(1), 82-96. http:// doi.org/10.1509/jppm.10.086

Muniady, R., Al- Mamun, A., Permarupan, P. Y., \& Zainol, N. R. B. (2014). Factors Influencing Consumer Behavior: A Study among University Students in Malaysia. Asian Social Science, 10(9), 18-25. http://doi.org/10.5539/ass.v10n9p18

Närvänen, E., Kartastenpää, E., \& Kuusela, H. (2013). Online lifestyle consumption community dynamics: A practice based analysis. Journal of Consumer Behaviour, 12(5), 358-369.

Neme, S. (2013). Eficacia de tácticas de influencia basadas en la coherencia, el compromiso y los significados culturales para el favorecimiento en la intención de conducta proambiental asociada al consumo de servicios públicos en hogares bogotanos. (Tesis inédita de Maestría). Fundación Universitaria Konrad Lorenz. Bogotá, Colombia. 
Nepomuceno, M. V., \& Laroche, M. (2015). The impact of materialism and anti-consumption lifestyles on personal debt and account balances. Journal of Business Research, 68(3), 654-664. http://doi.org/10.1016/j.jbusres.2014.08.006

Nie, C., \& Zepeda, L. (2011). Lifestyle segmentation of US food shoppers to examine organic and local food consumption. Appetite, 57(1), 28-37. http://doi.org/10.1016/j. appet.2011.03.012

Niu, H.-J., Chiang, Y.-S., \& Tsai, H.-T. (2012). An Exploratory Study of the Otaku Adolescent Consumer. Psychology \& Marketing, 29(10), 712-725. http://doi. org/10.1002/mar.20558

Onozaka, Y., Hansen, H., \& Sørvig, A. (2014). Consumer Product Perceptions and Salmon Consumption Frequency: The Role of of Heterogeneity Based on Food Lifestyle Segments. Marine Resource Economics, 29(4), 351-374. http://doi. org/10.1086/678928

Pagalea, A., \& Uta, D. S. V. (2012). Romanian Consumer Lifestyle and Attitude towards Bio Products Purchase. Procedia - Social and Behavioral Sciences, 62, 1308-1312. http://doi.org/10.1016/j.sbspro.2012.09.224

Pan, Y., Luo, L., Liu, D., Gao, L., Xu, X., Shen, W., \& Gao, J. (2014). How to Recommend by Online Lifestyle Tagging (OLT). International Journal of Information Technology \& Decision Making, 13(06), 1183-1209. http://doi.org/10.1142/ S0219622014500795

Park, S.-B., Lee, Y.-K., \& Chung, N. (2013). Why don't consumers go internet shopping in Korea? Segmentation of consumer. Behaviour and Information Technology, 32(5), 468-479. http://doi.org/10.1080/0144929X.2012.687771

Parris, D. L., Shapiro, S. L., Welty Peachey, J., Bowers, J., \& Bouchet, A. (2015). More than competition: exploring stakeholder identities at a grassroots cause-related sporting event. International Review on Public and Nonprofit Marketing, 12(2), 115-140. http://doi.org/10.1007/s12208-015-0129-4

Petticrew, M. y Roberts, H. (2008). Systematic reviews in the social sciences: A practical guide. Nueva York: Wiley.

Qing, P., Lobo, A., \& Chongguang, L. (2012). The impact of lifestyle and ethnocentrism on consumers' purchase intentions of fresh fruit in China. Journal of Consumer Marketing, 29(1), 43-51. http://doi.org/10.1108/07363761211193037

Rahulan, M., Troynikov, O., Watson, C., Janta, M., \& Senner, V. (2013). Consumer Purchase Behaviour of Sports Compression Garments -A study of Generation Y and Baby Boomer Cohorts. Procedia Engineering, 60, 163-169. http://doi.org/10.1016/j. proeng.2013.07.055

Rong-Da Liang, A. (2014). Enthusiastically consuming organic food. Internet Research, 24(5), 587-607. http://doi.org/10.1108/IntR-03-2013-0050

Rosenberg, B. C. (2012). Dangerous Houses: Scientific Lifestyle Television and Risk Management. Home Cultures, 9(2), 173-194. http://doi.org/10.2752/17517421 2X13325123562269

Salome, L., \& van Bottenburg, M. (2012). Are they all daredevils? Introducing a participation typology for the consumption of lifestyle sports in different settings. European Sport Management Quarterly, 12(1), 19-42. http://doi.org/10.1080/161 84742.2011.637171 
Sanabria Torres, E., \& Parra Penagos, C. O. (2013). Caracterización del comprador sogamoseño en súper e hipermercados. Estudios Gerenciales, 29(126), 49-57. http:// doi.org/10.1016/S0123-5923(13)70019-6

Santisi, G., Platania, S., \& Hichy, Z. (2014). A lifestyle analysis of young consumers: a study in Italian context. Young Consumers, 15(1), 94-104. http://doi.org/10.1108/ YC-03-2013-00357

Sarabia, F., Dolores De Juan, M., y González, A. (2009). Valores y estilo de vida de los consumidores. Cómo entenderlos y medirlos. Madrid: Ediciones Pirámide.

Schäfer, M., Jaeger-Erben, M., \& dos Santos, A. (2011). Leapfrogging to Sustainable Consumption? An Explorative Survey of Consumption Habits and Orientations in Southern Brazil. Journal of Consumer Policy, 34(1), 175-196.

Scheerder, J., Vos, S., \& Taks, M. (2011). Expenditures on Sport Apparel: Creating Consumer Profiles through Interval Regression Modelling. European Sport Management Quarterly, 11(3), 251-274. http://doi.org/10.1080/16184742.2011.577931

Schnettler, B., Miranda, H., Lobos, G., Sepúlveda, J., \& Denegri, M. (2011). A study of the relationship between degree of ethnocentrism and typologies of food purchase in supermarkets in central-southern Chile. Appetite, 56(3), 704-12. http://doi. org/10.1016/j.appet.2011.02.007

Siekierski, P., Ponchio, M. C., \& Strehlau, V. I. (2013). Influence of Lifestyles Related to Eating Habits in Ready Meal Consumption: comparative study between São Paulo and Rome. Review of Business Management, 15(48), 325-342. http://doi. org/10.7819/rbgn.v15i48.1223

Smith, A. (2010). Lifestyle television programmes and the construction of the expert host. European Journal of Cultural Studies, 13(2), 191-205. http://doi. org/10.1177/1367549409352279

Spillan, J. E., Kang, Z., \& Barat, S. (2011). A Multi-Country Study Exploring Relationships of Lifestyles to Ethnocentrism. Journal of Transnational Management, 16(2), 62-83. http://doi.org/10.1080/15475778.2011.571609

Strizhakova, Y., \& Coulter, R. A. (2013). The "green" side of materialism in emerging BRIC and developed markets: The moderating role of global cultural identity. International Journal of Research in Marketing, 30(1), 69-82. http://doi. org/10.1016/j.jiresmar.2012.08.003

Suresh, S., \& Ravichandran, S. (2010). Using Lifestyle Analysis to Develop Wellness Marketing Strategies for IT Pr...: EBSCOhost. Health Marketing Quarterly, 27(1), 1-20. http://doi.org/10.1080/07359680903263680

Szerényi, Z. M., Ágnes, Z., \& Anna, S. (2011). Consumer behaviour and lifestyle patterns of Hungarian students with regard to environmental awareness. Society and Economy, 33(1), 89-109. http://doi.org/10.1556/SocEc.33.2011.1.8

Tan, Y., Shaw, P., Cheng, H., \& Kim, K. K. (2013). The Construction of Masculinity: A Cross-Cultural Analysis of Men's Lifestyle Magazine Advertisements. Sex Roles, 69(5-6), 237-249. http://doi.org/10.1007/s11199-013-0300-5

Thongplew, N., van Koppen, C. S. A. (Kris), \& Spaargaren, G. (2014). Companies contributing to the greening of consumption: findings from the dairy and appliance industries in Thailand. Journal of Cleaner Production, 75, 96-105. http://doi. org/10.1016/j.jclepro.2014.03.076 
Unčovská, J., \& Ulčák, Z. (2015). ORGANIC BOX SCHEMES: FASHION OR DOWNSHIFTING? Agriculture \& Forestry/Poljoprivreda I Sumarstvo, 61(1), 59-66. http://doi.org/10.17707/AgricultForest.61.1.07

Valentine, D., \& L. Powers, T. (2013). Generation Y values and lifestyle segments. Journal of Consumer Marketing, 30(7), 597-606. http://doi.org/10.1108/JCM-072013-0650

Veal, A. (1993). The concept of lifestyle: a review. Leisure Studies, 12(4), 233-252.

Vecchio, R., \& Annunziata, A. (2015). Willingness-to-pay for sustainability-labelled chocolate: an experimental auction approach. Journal of Cleaner Production, 86, 335-342. http://doi.org/10.1016/j.jclepro.2014.08.006

Wang, J. J., Zhao, X., \& Li, J. J. (2013). Group Buying: A Strategic Form of Consumer Collective. Journal of Retailing, 89(3), 338-351. http://doi.org/10.1016/j.jretai.2013.03.001

Wicker, P., Hallmann, K., Prinz, J., \& Weimar, D. (2012). Who takes part in triathlon? An application of lifestyle segmentation to triathlon participants. International Journal of Sport Management and Marketing, 12(1/2), 1-29. http://doi.org/10.1504/ IJSMM.2012.051246

Woermann, N. (2012). On the Slope Is on the Screen: Prosumption, Social Media Practices, and Scopic Systems in the Freeskiing Subculture. American Behavioral Scientist, 56(4), 618-640. http://doi.org/10.1177/0002764211429363

Woermann, N., \& Rokka, J. (2015). Timeflow: How Consumption Practices Shape Consumers' Temporal Experiences. Journal of Consumer Research, 41(6), 1486-1508. http://doi.org/10.1086/680668

Yeh, N. C., \& Chen, Y. J. (2011). On the everyday life information behavior of LOHAS consumers: A perspective of lifestyle. Journal of Educational Media and Library Science, 48(4), 489-510. Retrieved from http://www.scopus.com/inward/record. url?eid=2-s2.0-80053095426\&partnerID=tZOtx3y1

Zepeda, L., \& Nie, C. (2012). What are the odds of being an organic or local food shopper? Multivariate analysis of US food shopper lifestyle segments. Agriculture and Human Values, 29(4), 467-480. http://doi.org/10.1007/s10460-012-9364-z

Zsóka, Á., Szerényi, Z. M., Széchy, A., \& Kocsis, T. (2013). Greening due to environmental education? Environmental knowledge, attitudes, consumer behavior and everyday pro-environmental activities of Hungarian high school and university students. Journal of Cleaner Production, 48, 126-138. http://doi.org/10.1016/j.jclepro.2012.11.030 